\title{
Modeling of Evaporation of Thin Films Using DOE
}

\author{
Pavel Mach, Miroslav Kocián \\ Department of Electrotechnology, Faculty of Electrical Engineering, Czech Technical University in Prague, \\ Prague, Czech Republic \\ mach@fel.cvut.cz
}

\begin{abstract}
Evaporation of thin films is widely spread technology. The goal of the work has been to optimize a process of fabrication of thin resistive Ni films. Mathematical models for calculation of thickness and resistance of the films have been created. A method of Design of Experiments has been used for design of the models. Three types of models have been designed and tested: a linear model, a combined one and a short combined one. First the models have been verified using an Ftest. It has been found that a linear model is not satisfactory, therefore it has been rejected. Subsequently remaining two models have been verified by complementary experiments, when calculated values and values measured on newly fabricated films have been compared. It has been found an excellent conformity among calculated and measured values ( $\pm 3 \%)$. Then the optimum fabrication conditions have been found by optimization of the models.
\end{abstract}

Keywords: evaporation, thin film, DOE, statistical testing

\section{Introduction}

Thin films are the basis for a large number of technologies and are one of the most important tools for the preparation of many different types of electronics components. Analysis of the growth and characterization of thin films is an important subject for basic as well as applied research. Important properties of thin films are strongly affected by technology of their fabrication. Therefore great effort is paid to analysis of processes of thin film fabrication.

A number of different types of fabrication processes for thin film coatings are being intensively studied. The number of types of these processes is very high. Processes based on physical vapor deposition (PVD) as well as processes based on chemical vapor deposition (CVD) belong to them.

Basic types of PVD processes are vacuum evaporation and vacuum sputtering. Evaporation is used for fabrication of films, whose density is comparable with the density of balk of evaporated material, whereas sputtering is often used for fabrication of low density films. Physical properties of evaporated and sputtered films differ, especially as for their mechanical properties.
Evaporation is not a new technology. There are many modifications of this process, which is contemporary used in many different applications. The goal of the paper is to describe the process of evaporation of thin $\mathrm{Ni}$ films using a mathematical model with sufficient accuracy.

There are different ways how to find a mathematical model of a technological process. With respect to our experience in the field of DOE this method has been chosen.

\section{Evaporation and measurement of thin films}

Mathematical model of evaporation has been searched with the aim to optimize the process of fabrication of $\mathrm{Ni}$ resistors. $\mathrm{Ni}$ (99.99) has been evaporated on glass substrates. Topology of the resistors is shown in Fig. 1, four resistors have been fabricated on one substrate.

Thin films are evaporated at the pressure of $10 \mathrm{mPa}$ or lower. The lower is the pressure; the higher is quality of fabricated films. Evaporation equipment has been pumped by two rotary pumps and one diffusion 
pump. Evaporation has been carried out at the pressure of $2 \mathrm{mPa}$. The schematic diagram of this equipment is shown in Fig. 2.

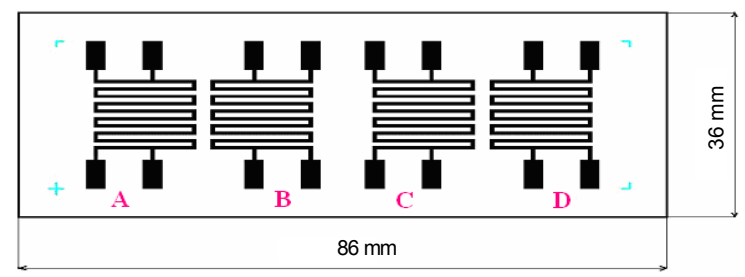

Fig. 1 Topology of fabricated resistors

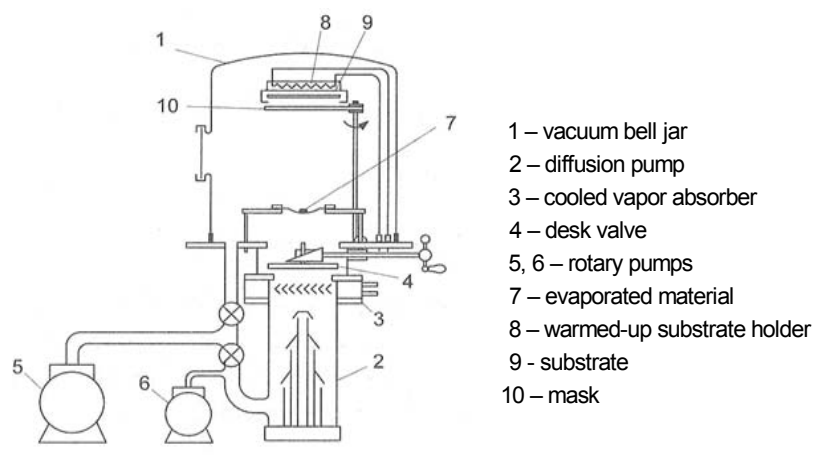

Fig. 2 Schematic diagram of evaporator

Two parameters of fabricated films have been investigated:

- The film thickness.

- The resistance of the resistors.

These parameters have been optimized depending on following parameters of the technological process:

- The mass of the evaporated material.

- The pressure.

- The temperature of the substrate.

Every technological parameter (technological factor) has bee used at two levels (see Tab. 1)

\begin{tabular}{|lcc|}
\hline Parameter of the process & $\begin{array}{c}\text { Lower } \\
\text { limit }\end{array}$ & $\begin{array}{c}\text { Upper } \\
\text { limit }\end{array}$ \\
\hline Evaporated mass $(\mathrm{mg})$ & $\mathbf{7 0}$ & $\mathbf{1 4 0}$ \\
Pressure $(\mathrm{mPa})$ & $\mathbf{2 , 5}$ & $\mathbf{6 , 5}$ \\
Temperature of the substrate $\left({ }^{\circ} \mathrm{C}\right)$ & $\mathbf{3 0}$ & $\mathbf{1 3 0}$ \\
\hline
\end{tabular}

Tab. 1 Limits of technological factors A, B and C

A ... evaporated mass $(\mathrm{mg}), \mathrm{B} \ldots$ pressure $(\mathrm{Pa}), \mathrm{C} \ldots$ temperature of the substrate $\left({ }^{\circ} \mathrm{C}\right)$
The thickness of the films has been measured by the use of Talystep (Taylor Hobson, type EP 43, see Fig. 3), the equipment has been completed by a program for automatic evaluation of measured thickness. The resistance of the resistors has been measured using HP 34401A Multimeter (Hewlett Packard). Tungsten test prods have been used for the measurement of the resistors.

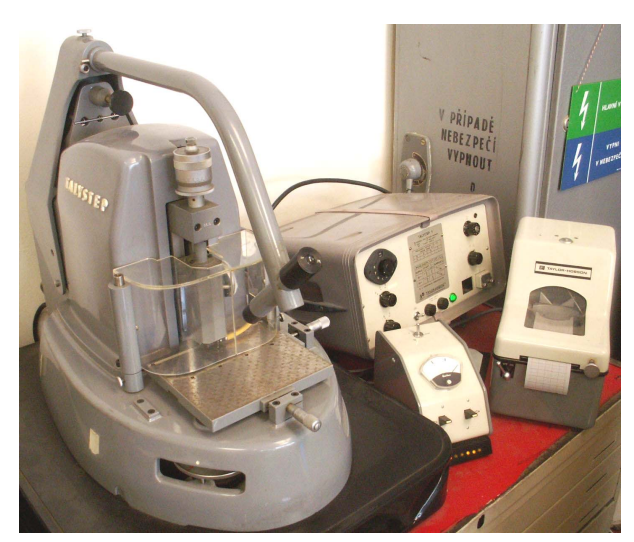

Fig. 3 Talystep

The total number of the measured resistors has been 96 for every combination of technological factors and their levels.

\section{Design of Experiments}

A full plan of experiments of the type $2^{3}$ has been chosen for design of a mathematical model of the process [1], [2]. The schematic diagram of this type of experiments is shown in Tab. 2.

First estimations of influences of factors and their interactions have been calculated. Following formulas have been chosen for calculations:

$$
\begin{aligned}
& Z_{A}=a+a c+a b+a b c-(1)-c-b-b c \\
& Z_{B}=b+b c+a b+a b c-(1)-c-a-a c \\
& Z_{C}=c+b c+a c+a b c-(1)-b-a-a b \\
& Z_{A B}=a b+a b c+(1)+c-a-a c-b-b c \\
& Z_{A C}=a c+a b c+(1)+b-a-a b-c-b c \\
& Z_{B C}=b c+a b c+(1)+a-c-a c-b-a \\
& Z_{A B C}=a+b+c+a b c-a b-a c-b c-(1)
\end{aligned}
$$




\begin{tabular}{|c|c|c|c|c|c|c|c|}
\hline \multicolumn{4}{|c|}{$A_{1}$} & \multicolumn{4}{|c|}{$A_{2}$} \\
\hline \multicolumn{2}{|c|}{$B_{1}$} & \multicolumn{2}{|c|}{$B_{2}$} & \multicolumn{2}{|c|}{$\boldsymbol{B}_{1}$} & \multicolumn{2}{|c|}{$B_{2}$} \\
\hline $\begin{array}{c}C_{1} \\
A_{1} B_{1} C_{1}\end{array}$ & $\begin{array}{c}C_{2} \\
A_{1} B_{1} C_{2}\end{array}$ & $\begin{array}{c}C_{1} \\
A_{1} B_{2} C_{1}\end{array}$ & $\begin{array}{c}C_{2} \\
A_{1} B_{2} C_{2}\end{array}$ & $\begin{array}{c}C_{1} \\
A_{2} B_{1} C_{1}\end{array}$ & $\begin{array}{c}C_{2} \\
A_{2} B_{1} C_{2}\end{array}$ & $\begin{array}{c}C_{1} \\
A_{2} B_{2} C_{1}\end{array}$ & $\begin{array}{c}C_{2} \\
A_{2} B_{2} C_{2}\end{array}$ \\
\hline (1) & $c$ & $b$ & $b c$ & $a$ & $a c$ & $a b$ & $a b c$ \\
\hline$y_{l, 1}$ & $y_{2,1}$ & $y_{3,1}$ & $y_{4,1}$ & $y_{5,1}$ & $y_{6,1}$ & $y_{7,1}$ & $y_{8,1}$ \\
\hline$y_{1,2}$ & $y_{2,2}$ & $y_{3,2}$ & $y_{4,2}$ & $y_{5,2}$ & $y_{6,2}$ & $y_{7,2}$ & $y_{8,2}$ \\
\hline . & . & . & . & . & . & . & . \\
\hline . & . & . & . & . & . & . & . \\
\hline . & . & . & . & . & . & . & . \\
\hline$y_{l, r}$ & $y_{2, r}$ & $y_{3, r}$ & $y_{4, r}$ & $y_{5, r}$ & $y_{6, r}$ & $y_{7, r}$ & $y_{8, r}$ \\
\hline$R_{I}$ & $R_{2}$ & $R_{3}$ & $R_{4}$ & $R_{5}$ & $R_{6}$ & $R_{7}$ & $R_{8}$ \\
\hline
\end{tabular}

Tab. 2 Table of factorial experiments of the type $2^{3}$

Symbolic labelings of columns ((1), a, b, .., abc) is substituted by the sum of a relevant column $\left(R_{1}, R_{5}\right.$, $\mathrm{R}_{3}, \ldots, \mathrm{R}_{8}$ ) for numeric calculations.

Statistical significance of these estimations has been tested using an F-test. Following test characteistics have been calculated:

$$
\begin{aligned}
& F_{A}=\frac{S_{A}}{\frac{S_{r}}{v}}, \quad F_{B}=\frac{S_{B}}{\frac{S_{r}}{v}}, \\
& \cdots \ldots . \\
& F_{A B C}=\frac{S_{A B C}}{\frac{S_{r}}{v}}
\end{aligned}
$$

Where sums of squares:

$$
\begin{aligned}
& S_{A}=\frac{Z_{A}{ }^{2}}{d \cdot r}, \quad S_{B}=\frac{Z_{B}^{2}}{d \cdot r}, \\
& \ldots \ldots . \\
& S_{A B C}=\frac{Z_{A B C}^{2}}{d \cdot r}
\end{aligned}
$$

Rezidual sum of squares is calculated using a formula:

$$
S_{r}=\sum_{i=1}^{d} \sum_{j=1}^{r} y_{i, j}^{2}-\frac{1}{r} \sum_{i=1}^{d}\left(\sum_{j=1}^{r} y_{i, j}\right)^{2}
$$

Degrees of freedom have been calculated of an equation: $v=d \cdot(r-1)$

Calculated values of the test characteristics $\mathrm{F}_{\mathrm{A}}, \mathrm{F}_{\mathrm{B}}, \ldots$, $\mathrm{F}_{\mathrm{ABC}}$ have been compared with the value of $\mathrm{F}_{\alpha}(1, v)$ quantil, which has been found in statistical tables. If the value of calculated $\mathrm{F}$-characteristic has been higher than the value of the quantil, the parameter (or interaction of parameters), for which this test value has been calculated, has been involved into the mathematical model of the evaporation process. If the value of the test characteristic has been lower, the parameter (or interaction) has not been involved into the model.

The coefficients of the model have been calculated using the formulas:

$b_{0}=\frac{1}{d} \sum_{i=1}^{d} \bar{y}_{i}, \quad b_{1}=\frac{Z_{A}}{d \cdot r}$,

$b_{1,2,3}=\frac{Z_{A B C}}{d \cdot r}$

The mathematical model has been of a following structure:

$$
\begin{aligned}
& \tilde{y}=b_{0}+b_{1} x_{1}+b_{2} x_{2}+\ldots+b_{n} x_{n}+. \\
& . .+b_{12} x_{1} x_{2}+\ldots b_{123} x_{1} x_{2} x_{3}
\end{aligned}
$$

Where $\mathrm{x}_{1} \ldots$ transformed parameter $\mathrm{A}, \mathrm{x}_{2} \ldots$ transformed parameter $\mathrm{B}, \mathrm{x}_{3} \ldots$ transformed parameter 
C. Following equation has been used for transformation:

$$
\begin{aligned}
& X_{1}=\frac{2}{A_{2}-A_{1}} \cdot\left(A-\frac{A_{1}+A_{2}}{2}\right) \\
& X_{2}=\frac{2}{B_{2}-B_{1}} \cdot\left(B-\frac{B_{1}+B_{2}}{2}\right) \\
& X_{3}=\frac{2}{C_{2}-C_{1}} \cdot\left(C-\frac{C_{1}+C_{2}}{2}\right)
\end{aligned}
$$

Three types of the models have been found for calculation of the thickness $d$ of the evaporated films:

A linear one for calculation of $\mathrm{d}(\mathrm{nm})$ :

$\widetilde{y}=112,21+18,25 x_{1}-17,51 x_{2}+9,63 x_{3}$

A combined one for calculation of $\mathrm{d}(\mathrm{nm})$ :

$$
\begin{aligned}
& \tilde{y}=112,21+18,25 x_{1}-17,51 x_{2}+9,63 x_{3}+ \\
& +6,35 x_{1} x_{2}-4,01 x_{1} x_{3}+0,31 x_{2} x_{3}+0,1 x_{1} x_{2} x_{3}
\end{aligned}
$$

A contracted one for calculation of $\mathrm{d}(\mathrm{nm})$ :

$$
\begin{aligned}
\widetilde{y} & =12,21+18,25 x_{1}-17,51 x_{2}+9,63 x_{3}+ \\
& +6,35 x_{1} x_{2}-4,01 x_{1} x_{3}
\end{aligned}
$$

Quality of these models has been tested using the Ftest.

Contraction of the model has been carried out according to the results of the statistical testing. Significance of influence of factors and interactions has been tested.

After inverse transformation following models have been found:

A linear model for calculation of $\mathrm{d}(\mathrm{nm})$ :

$d=81,45+0,53 A-8,76 B+0,19 C$

A combined model for calculation of $\mathrm{d}(\mathrm{nm})$ :

$$
\begin{aligned}
& d=105,1+0,32 A-18,31 B+0,44 C+0,09 A B- \\
& -0,002 A C+0,0001 B C+0,00003 A B C
\end{aligned}
$$

A contracted model for calculation of $d(n m)$ :

$$
\begin{aligned}
& d=105,06+0,31 A-18,29 B+0,43 C+ \\
& +0,09 A B-0,002 A C
\end{aligned}
$$

The same procedure has been used for derivation of the models for the resistance $\mathrm{R}(\Omega)$ of the evaporated films.

A linear model for calculation of $\mathrm{R}(\Omega)$ :

$R=952,52-4,27 A+69,88 B-1,49 C$

A combined model for calculation of $\mathrm{R}(\Omega)$ :

$R=801,85-2,79 A+134,44 B-3,88 C-$

$-0,62 A B+0,03 A C+0,13 B C-0,001 A B C$

A contracted model for calculation of $\mathrm{R}(\Omega)$ :

$R=756,05-2,37 A+144,61 B-3,24 C-$

$-0,72 A B+0,02 A C$

Quality of the models has been tested using the F-test. Following results have been found:

a) models for calculation of the thickness of the evaporated films:

\begin{tabular}{|l|c|}
\hline Type of the model & $\begin{array}{c}\text { Result of } \\
\text { the } \boldsymbol{F} \text {-test }\end{array}$ \\
\hline Linear & complied \\
\hline Combined & complied \\
\hline Contracted & complied \\
\hline
\end{tabular}

Tab. 3 Testing of the models for calculation of the thickness of the films

b) models for calculation of the resistance of the evaporated films:

\begin{tabular}{|l|c|}
\hline Type of the model & $\begin{array}{c}\text { Result of } \\
\text { the } \boldsymbol{F} \text {-test }\end{array}$ \\
\hline Linear & $\begin{array}{c}\text { non- } \\
\text { complied }\end{array}$ \\
\hline Combined & complied \\
\hline Contracted & complied \\
\hline
\end{tabular}

Tab. 4 Testing of the models for calculation of the resistance of the films 
The profiles of the models have been simulated using Matlab [3]. Two examples of these simulations are

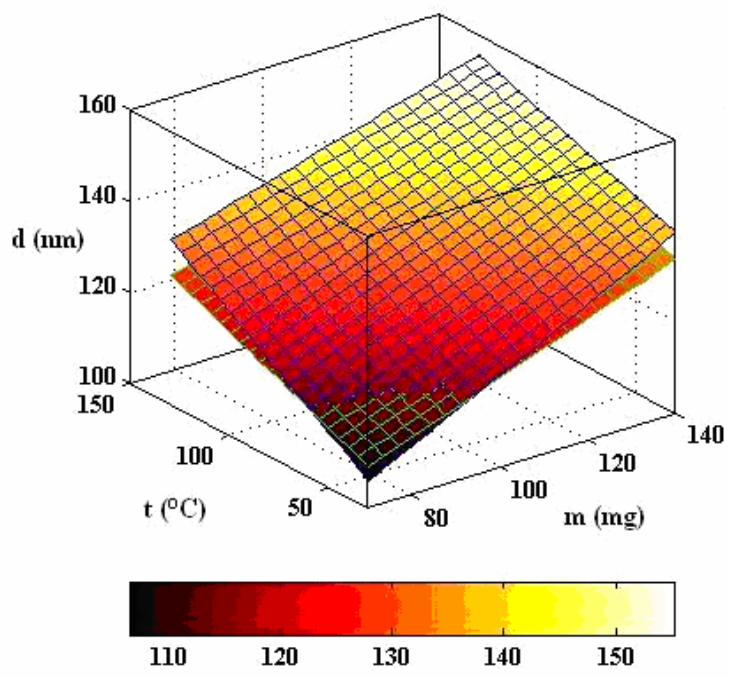

Fig. 4 Thickness of the evaporated film as a function of the temperature $t$ and mass $m$ of the evaporated material calculated using the linear (bottom) and combined (top) mathematical models for the pressure of $2.5 \mathrm{mPa}$

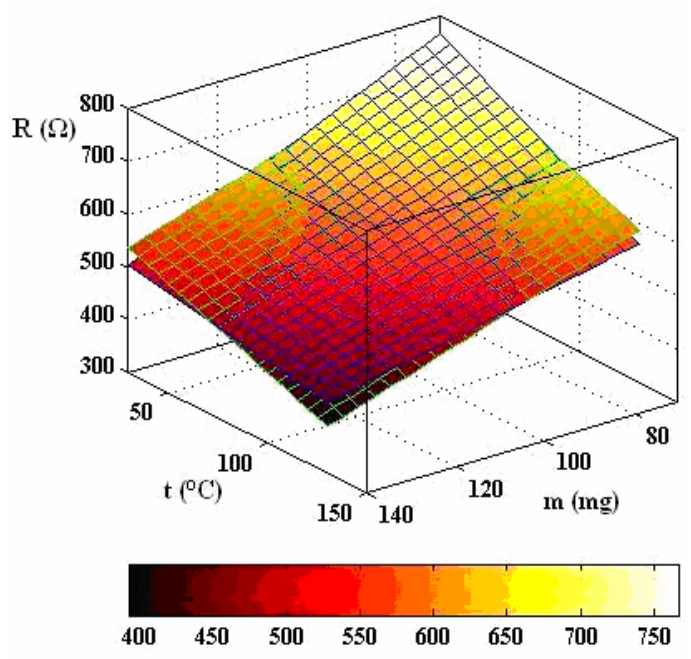

Fig. 4 Resistance of the evaporated film as a function of the temperature $t$ and mass $m$ of the evaporated material calculated using the linear (bottom) and combined (top) mathematical models for the pressure of $2.5 \mathrm{mPa}$ presented in following figures.

\section{Accuracy of the models}

Accuracy is the basic property of the model. Therefore accuracy of every model has been investigated such the way that complementary experiments have been carried out inside the area of binding limits. Measured values of the thickness and resistances of every film have been compared with the values calculated of the models. The results have been as follows: the maximum difference between the value of the thickness of the evaporated film and the value calculated of the model has been up to $15 \%$ for the linear model, up to $5 \%$ for the contracted model and lower than $2 \%$ for the combined model. The maximum difference between the value of the resistance of evaporated film and the value calculated of the model has been $2,5 \%$ for the contracted model and lower than $1 \%$ the combined model. The linear model has not passed the F-test.

\section{Conclusions}

DOE has been used for formation of mathematic models for calculation of the thickness and the resistance of evaporated Ni films. Three types of the models have been calculated: the linear one, the combined one and the contracted one. All models have been constructed over the same measured data. The best results have been obtained by the use of combined models. Differences of the values of thickness and the resistance calculated of the models and measured on fabricated layers have been lower than $2 \%$.

It has been found that DOE is an efficient method for simulation of technological processes.

\section{References}

[1] Montgomery, D. C.: Introduction to Statistical Quality Control. J Willey and Sons, Inc. New York. 796 pp. 2001

[2] Breyfogle III, W. F.: Implementing Six Sigma. J. Willey and Sons, Inc. New York. 791 pp. 1999

[3] Matlab - User's Manual

\section{Acknowledgment}

The work has been supported by a research project $\mathrm{Nr}$. 34/05212/13113 "Diagnostics of materials". 